\title{
Bone marrow fibroblasts in patients with advanced lung cancer
}

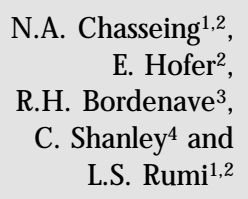

N.A. Chasseing ${ }^{1,2}$, E. Hofer ${ }^{2}$,

R.H. Bordenave3,

C. Shanley 4 and L.S. Rumi ${ }^{1,2}$

\author{
${ }^{1}$ Research Member of the Consejo Nacional de Investigaciones Científicas y \\ Técnicas de la República Argentina (CONICET), Buenos Aires, Argentina \\ ${ }^{2}$ Instituto de Biología y Medicina Experimental, Buenos Aires, Argentina \\ ${ }^{3}$ Departamento de O ncologia, I. Iriarte Hospital, Buenos Aires, Argentina \\ ${ }^{4}$ Departamento de Hematologia e Transplante de Médula Ó ssea, \\ Hospital Británico, Buenos Aires, Argentina
}

\section{Correspondence \\ N.A. Chasseing \\ Instituto de Biología y \\ Medicina Experimental \\ Vuelta de O bligado, 2490 \\ 1428 Buenos Aires \\ Argentina \\ Fax: +54-11-4786-2564 \\ E-mail: chaseing@ibyme.dna.uba.ar}

Some of these data were presented at the XLIII Reunión Anual de la Sociedad Argentina de Investigación Clínica (SAIC), Mar del Plata,

Argentina, November 26-29, 1998.

Research supported by CONICET (PEI No. 0098/97) and the Roemmers Foundation.

Received May 24, 2001

Accepted August 27, 2001

\section{Abstract}

In a previous study we demonstrated that the incidence of fibroblast colony-forming units (CFU-F) was very low in bone marrow primary cultures from the majority of untreated advanced non-small lung cancer patients (LCP) compared to normal controls (NC). For this reason, we studied the ability of bone marrow stromal cells to achieve confluence in primary cultures and their proliferative capacity following four continuous subcultures in consecutive untreated LCP and NC. We also evaluated the production of interleukin-1B (IL-1ß) and prostaglandin E2 (PGE2) by pure fibroblasts. Bone marrow was obtained from $20 \mathrm{LCP}$ and $20 \mathrm{NC}$. A CFU-F assay was used to investigate the proliferative and confluence capacity. Levels of IL-1ß and PGE2 in conditioned medium (CM) of pure fibroblast cultures were measured with an ELISA kit and RIA kit, respectively. Only fibroblasts from 6/13 (46\%) LCP confluent primary cultures had the capacity to proliferate following four subcultures $(\mathrm{NC}=100 \%)$. Levels of spontaneously released IL-1ß were below $10 \mathrm{pg} / \mathrm{ml}$ in the CM of LCP, while NC had a mean value of 1,217 $\pm 74 \mathrm{pg} / \mathrm{ml}$. In contrast, levels of PGE2 in these CM of LCP were higher (77.5 \pm 23.6 $\mathrm{pg} / \mathrm{ml})$ compared to $\mathrm{NC}(18.5 \pm 0.9 \mathrm{pg} / \mathrm{ml})$. In conclusion, bone marrow fibroblasts from LCP presented a defective proliferative and confluence capacity, and this deficiency may be associated with the alteration of IL-1ß and PGE2 production.

\section{Key words}

- Fibroblasts

- Interleukin-1

- Prostaglandin E2

- Cancer

\section{Introduction}

Several in vitro studies of the hematopoietic microenvironment using long-term bone marrow and fibroblast colony-forming units (CFU-F) culture systems have proved that most (60-90\%) adherent stromal cells are fibroblasts (1-3). Reports also indicate that normal human bone marrow fibroblasts ca- pable of producing CFU-F and the conditioned medium $(\mathrm{CM})$ released by them can support proliferation and differentiation of committed progenitors of the myeloid and erythroid series as well as stromal cells in liquid culture systems (4-6). In previous studies, we demonstrated that CFU-F incidence was very low or that clusters were the only or the predominant type of clonal growth in 
primary bone marrow cultures from the majority of untreated advanced non-small lung cancer patients (LCP) (7). This reduction in the number of CFU-F may be related to an increase in the levels of inhibitor factors and/or to a decrease of the levels of stimulant mediators such as interleukin-1 (IL-1), IL17 , platelet derived growth factor, transforming growth factor- $\$ 1$ (TGF- $\$ 1$ ), epidermal growth factor, tumor necrosis factor receptor, collagen, fibronectin, IL-2, interferon- $\gamma$, and prostaglandin E2 (PGE2) (8-13). For this reason, we have evaluated the ability of bone marrow stromal cells (mostly fibroblasts) to achieve confluence in primary cultures and their proliferative capacity following four continuous subcultures in consecutive untreated LCP and normal controls (NC). At the same time, we studied the release of IL-1ß and PGE2 into CM of pure fibroblast cultures treated or not with muramyl-dipeptide (MDP).

\section{Material and Methods}

\section{Patients}

Bone marrow samples were obtained from $20 \mathrm{NC}$ and 20 consecutive untreated patients with non-small lung epidermoid carcinoma (stage III A, III B and IV). We used a UICC TNM classification. All LCP and NC were age and sex matched. The age intervals were: $\mathrm{LCP}=40$ to 60 and $\mathrm{NC}=45$ to 67 years. All individuals gave consent to participate in these studies, which were performed in accordance with the principles of the Declaration of Helsinki. Patient bone marrow aspirates were provided by Dr. R.H. Bordenave, Department of Oncology, I. Iriarte Hospital, Buenos Aires, Argentina. Healthy control bone marrow aspirates were provided by Dr. C. Shanley, Department of Hematology and Bone Marrow Transplantation, Británico Hospital, Buenos Aires, Argentina. NC were healthy donors for bone marrow transplantation.

The present investigation was approved by the Británico Hospital and I. Iriarte Hospital Ethics Committees.

\section{Bone marrow micrometastases}

Bone marrow infiltration with neoplastic cells was detected by immunocytochemistry staining (biotin-streptavidin-peroxidase, Universal Dako LSAB System, Carpinteria, CA, USA) and an analysis of cell morphology was done by the Pappenheim technique. Bone marrow samples were stained with monoclonal antibodies to cytokeratin AE1-AE3 (Dako), cytokeratin 7 (CK7, Dako) and cytokeratin 20 (CK20, Dako). Patients were considered positive for micrometastasis only if cells expressed cytokeratin AE1-AE3, CK7 and/or CK20 and if these were morphologically malignant.

\section{Collection and preparation of bone marrow cells}

Bone marrow samples were collected under local anesthesia from the posterior iliac crest into heparinized saline without preservatives ( 25 units $/ \mathrm{ml}$, Gibco, Grand Island, NY, USA). Aspirates were diluted 1/2 with PBS, $\mathrm{pH} 7.5$, and were layered onto Histopaque (density $=1,075 \mathrm{~g} / \mathrm{cm}^{3}$; Sigma, St. Louis, MO, USA). After being centrifuged for $25 \mathrm{~min}$ at $1,500 \mathrm{rpm}$, mononuclear cells were harvested from the interface, washed twice in PBS and resuspended in $\alpha$ medium (Gibco) containing $100 \mathrm{IU} / \mathrm{ml}$ penicillin (Gibco), $100 \mu \mathrm{g} / \mathrm{ml}$ streptomycin (Gibco) and $25 \mu \mathrm{g} / \mathrm{ml}$ of amphotericin B (Gibco). The cell suspension was counted with $3 \%$ acetic acid solution and cell viability was determined by Trypan blue exclusion.

\section{Fibroblast colony-forming units assay}

Viable, light density mononuclear cells $\left(5 \times 10^{6}\right)$ were placed in $25-\mathrm{cm}^{2}$ tissue culture flasks (Corning) which contained $10 \mathrm{ml}$ 
of supplemented previously described $\alpha$-medium and 20\% heat-inactivated FBS (Gibco cat No. 16,000-044) (1). The cells were incubated at $37^{\circ} \mathrm{C}$ in a $5 \% \mathrm{CO}_{2}$ humidified environment for 7 days. After this period, the non-adherent cells were removed and the medium was renewed. Then the primary cultures were returned to incubation for an additional 7 days. At the end of this period, the medium was discarded and the adherent cells were washed twice with PBS, fixed with $100 \%$ methanol, and stained with Giemsa. Clones of $>50$ cells were scored as CFU-F under a binocular microscope.

\section{Confluent primary culture formation}

Viable, light density mononuclear cells $\left(5 \times 10^{6}\right)$ of each sample were placed in 25$\mathrm{cm}^{2}$ tissue culture flasks (Corning) which contained $10 \mathrm{ml}$ of supplemented previously described $\alpha$-medium and $20 \%$ FBS. This supplemented medium is known to be selective for fibroblast progenitor proliferation (1). The cells were incubated at $37^{\circ} \mathrm{C}$ in a $5 \%$ $\mathrm{CO}_{2}$ humidified environment for 7 days. After this period, the non-adherent cells were removed and the medium was renewed. This period of time was selected in order to allow the maximum release of growth factors, which are necessary for fibroblast progenitor proliferation (1). The primary cultures were returned to incubation for further days until confluence. From the initiation of the experiment until day 60, or until the cells reached confluence, the medium was changed every 7 days. At the end, the medium was discarded and the confluent adherent cells were washed twice with PBS and then trypsinized with a solution of trypsin-EDTA (0.05-0.02\% in PBS, respectively; Gibco). Finally, trypsin-sensitive adherent cells were further induced to proliferate following four continuous subcultures. The adherent cells were subcultured only after confluence in each case. The number of days the adherent cells (most of them fibroblasts) took to achieve confluence in primary cultures was studied, as well as the number of patients with fibroblast proliferative capacity when cells from confluent primary cultures were further induced to proliferate following four subcultures.

The fibroblastic nature of the adherent cells that composed the cultures was demonstrated by immunofluorescent staining with monoclonal antibodies against human fibronectin (a gift from Dr. A. Kornbliht, Facultad de Ciencias Exactas y Naturales, Universidad de Buenos Aires, Argentina) and against the human $B$ subunit of prolyl-4hydroxylase (Dako). We also used cytochemical analyses with alkaline phosphatase stain. Adherent cells were fixed with 50\% methanol.

\section{Preparation of CM from pure fibroblasts}

Bone marrow fibroblasts isolated after four continuous confluent subcultures were adjusted to $5 \times 10^{4}$ viable cells $/ \mathrm{ml}$ in fresh $\alpha$ medium containing $20 \%$ FBS and $1 \%$ antibiotic-antimycotic agent. The fibroblasts were allowed to adhere to plastic tissue culture plates (6 wells/plate, Falcon) for at least $24 \mathrm{~h}$ after the fourth subculture in order to obtain confluence before the experiment began. After this period, fibroblasts were incubated in the presence and absence of MDP $(1 \mu \mathrm{g} /$ $\mathrm{ml}$, Sigma) for an additional $72 \mathrm{~h}$ at $37^{\circ} \mathrm{C}$ and $5 \% \mathrm{CO}_{2}$ (14). The $\mathrm{CM}$ were obtained by centrifugation at $1,000 \mathrm{rpm}$ for $10 \mathrm{~min}$ and frozen at $-20^{\circ} \mathrm{C}$ before use for IL- $1 \beta$ assay and at $-70^{\circ} \mathrm{C}$ for PGE2.

\section{Determination of IL-1ß and PGE2 in the CM of pure fibroblast cultures}

IL-1ß was measured with an ELISA kit developed and given to us by Dr. E. Eugui (Syntex Research, Palo Alto, CA, USA) and PGE2 was quantified by the RIA kit of Dupont. For both soluble factors, the CM were diluted 1:5. The IL-1ß assay detects 
levels between 10 and 7,400 pg/ml and the PGE2 assay detects levels between 2.5 and $250 \mathrm{pg} / \mathrm{ml}$.

Simultaneously we evaluated IL-1ß and PGE2 levels in samples of supplemented $\alpha$ medium after 7 days of incubation, and observed that the concentration for both soluble factors was below the minimum detectable dose. All samples and standards were analyzed in duplicate.

\section{Statistical analysis}

Statistical analyses were performed using parametric and nonparametric tests de-

Table 1. Evaluation of fibroblast colony-forming units and the ability of the stromal cells to achieve confluence in bone marrow primary cultures.

\begin{tabular}{|c|c|c|c|c|}
\hline \multirow[t]{2}{*}{ Samples } & \multicolumn{2}{|c|}{ LCP } & \multicolumn{2}{|c|}{ NC } \\
\hline & $\begin{array}{c}\text { CFU-F/ } \\
5 \times 10^{6} \mathrm{CMN}\end{array}$ & $\begin{array}{l}\text { Confluence in } \\
\text { primary cultures } \\
\text { (days) }\end{array}$ & $\begin{array}{c}\text { CFU-F/ } \\
5 \times 10^{6} \mathrm{CMN}\end{array}$ & $\begin{array}{l}\text { Confluence in } \\
\text { primary cultures } \\
\text { (days) }\end{array}$ \\
\hline 1 & 36 & $30 *$ & 33 & 15 \\
\hline 2 & 15 & $30 *$ & 57 & 14 \\
\hline 3 & 8 & $17 *$ & 46 & 20 \\
\hline 4 & 0 & $22 *$ & 44 & 16 \\
\hline 5 & 25 & $22 *$ & 42 & 15 \\
\hline 6 & 0 & 30 & 58 & 18 \\
\hline 7 & 0 & 30 & 45 & 18 \\
\hline 8 & 25 & 45 & 78 & 16 \\
\hline 9 & 38 & 47 & 62 & 20 \\
\hline 10 & 13 & 20 & 80 & 15 \\
\hline 11 & 10 & 56 & 85 & 20 \\
\hline 12 & 0 & No & 191 & 14 \\
\hline 13 & 0 & No & 88 & 18 \\
\hline 14 & 0 & No & 70 & 18 \\
\hline 15 & 25 & No & 69 & 20 \\
\hline 16 & 10 & $16 *$ & 33 & 18 \\
\hline 17 & 0 & No & 148 & 18 \\
\hline 18 & 0 & No & 60 & 17 \\
\hline 19 & 5 & No & 108 & 22 \\
\hline 20 & 10 & 56 & 78 & 27 \\
\hline $\bar{x} \pm$ SEM & $11.0 \pm 2.8^{a}$ & $32.4 \pm 3.9^{\mathrm{b} \#}$ & $73.8 \pm 8.7^{a}$ & $17.9 \pm 0.7^{b}$ \\
\hline
\end{tabular}

Data are reported as means of duplicate individual values. \#This mean was obtained from the patients who showed confluent adherent cells up to 60 days after the beginning of incubation. $\mathrm{LCP}=$ lung cancer patients; $\mathrm{NC}=$ normal controls; $\mathrm{CMN}=$ light density mononuclear cells; CFU-F = fibroblast colony-forming units; $\mathrm{No}=$ did not reach confluence within the first 60 days.

*Six LCP confluent primary cultures whose adherent cells could proliferate in the four subsequent subcultures compared to the $100 \%$ of NC.

aP $<0.0001$ and bP $<0.0004$ LCP vs NC (nonparametric Mann-Whitney test). pending on the data studied $(\mathrm{P}<0.05)$.

\section{Results}

\section{Bone marrow micrometastases}

Using the Pappenheim and immunocytochemistry techniques, morphological evidence of bone marrow infiltration with neoplastic cells was not observed in cancer patients.

\section{Evaluation of proliferative and confluence capacity of the adherent cells in bone marrow primary cultures and four continuous subcultures}

Table 1 shows that only 5/20 (25\%) bone marrow cultures from LCP had adherent cells which were able to achieve confluence in primary cultures within the normal range (14 to 27 days). Furthermore, in the other 15/ $20(75 \%)$ cultures from LCP we observed that there was lack of confluence (35\% of the total) or that more days were needed for the stromal layers to obtain confluence (40\% of the total). The values (mean \pm SEM) were: $\mathrm{LCP}=32.4 \pm 3.9$ days and $\mathrm{NC}=17.9 \pm 0.7$ days $(\mathrm{P}<0.0004$, nonparametric MannWhitney test).

In addition, after 14 days of incubation of bone marrow primary cultures, the number of CFU-F was very low (2/7) or zero (5/7) in the LCP samples with a lack of confluence. Moreover, all the cultures of adherent cells from LCP samples which were able to achieve confluence in primary cultures showed a significantly decreased number of CFU-F compared to the values for NC. This last observation was independent of whether or not the cultures were able to achieve confluence within the normal range (Table 1).

On the other hand, when adherent cells from confluent primary cultures of LCP (13/ 20) were further induced to proliferate following four continuous subcultures, we observed that the growth had diminished or 
stopped between the second and third subculture in seven LCP cultures. Moreover, a high percentage of these seven LCP cultures showed the longest times to achieve confluence in bone marrow primary cultures (Table 1). Regarding, the six LCP cultures that were able to proliferate and to reach confluence in each of the four continuous subcultures, the number of days they needed to achieve confluence in each of the four individual subcultures was always longer than for the NC subcultures, although we incubated the same amount of adherent cells in both groups. In contrast, all NC cultures presented full confluent stromal layers in primary cultures and had the capacity to proliferate following four continuous subcultures.

In terms of lineage markers, up to $90 \%$ of the non-hematopoietic adherent cells from patients and NC primary cultures expressed three fibroblast markers (fibronectin, prolyl4-hydroxylase and alkaline phosphatase). Moreover, the majority of adherent cells in primary cultures presented a fusiform shape in both groups.

Regarding the number of trypsin-EDTA (0.05-0.02\%)-sensitive adherent cells (pure fibroblasts) in the fourth confluent subculture, LCP values did not differ from NC values $\left[\mathrm{LCP}=(0.805 \pm 0.074) \times 10^{6}\right.$ and $\mathrm{NC}$ $\left.=(1.150 \pm 0.220) \times 10^{6}\right]$. It is well known that only adherent bone marrow cells that have a fibroblastic nature are detached after this treatment (1).

\section{Determination of IL-1ß and PGE2 levels in the $\mathrm{CM}$ of pure fibroblast cultures}

The levels of IL- $1 ß$ released in the CM of fibroblast cultures from LCP were below the detectable amount $(<10 \mathrm{pg} / \mathrm{ml})$, independently of the culture treatment used (Table 2). In contrast, the levels of IL-1ß were significantly higher in the $\mathrm{CM}$ of $\mathrm{NC}$.

Finally, the levels of PGE2 spontaneously released in the CM of fibroblast cultures from LCP increased significantly com- pared to the mean value obtained in $\mathrm{NC}$ cultures $(\mathrm{P}<0.0022$, nonparametric MannWhitney test; Table 2). Moreover, in the presence of MDP a lack of response was observed in all LCP cultures, but PGE2 levels increased in the $\mathrm{CM}$ of $\mathrm{NC}(\mathrm{P}<0.0022$ compared to basal levels, nonparametric Mann-Whitney test).

\section{Discussion}

Bone marrow stromal cells produce multiple factors (extracellular matrix and cytokines), which are not only capable of controlling the self-renewal, proliferation and differentiation of hematopoietic stem/progenitor cells, but are also regulated by them $(12,15)$. Functional alterations in the bone marrow microenvironment may be involved in the manifestation of some malignant disorders $(16,17)$.

The results presented in this paper show that a high percentage of bone marrow primary cultures from untreated LCP had phenotypic abnormalities such as diminished ability of the adherent stromal cells to achieve confluence or a lack of confluence at all. These observations are likely to reflect derangement in the composition of the hematopoietic microenvironment and/or in the pro-

Table 2. Determination of the levels of interleukin$1 ß$ (IL-1ß) and prostaglandin E2 (PGE2) in the conditioned media of pure fibroblast cultures.

\begin{tabular}{lcc}
\hline Groups & IL-1ß $(\mathrm{pg} / \mathrm{ml})$ & PGE2 $(\mathrm{pg} / \mathrm{ml})$ \\
\hline LCP & & \\
None & $<10$ & $77.5 \pm 23.6^{\mathrm{b}}$ \\
MDP $(1 \mu \mathrm{g} / \mathrm{ml})$ & $<10$ & $47.3 \pm 17.6$ \\
NC & & \\
None & $1,217 \pm 74^{\mathrm{a}}$ & $18.5 \pm 0.9^{\mathrm{b}, \mathrm{c}}$ \\
MDP $(1 \mu \mathrm{g} / \mathrm{ml})$ & $4,607 \pm 62^{\mathrm{a}}$ & $90.0 \pm 5.7^{\mathrm{c}}$
\end{tabular}

Data are reported as means \pm SEM. LCP = lung cancer patients; $\mathrm{NC}=$ normal controls; $\mathrm{MDP}=$ muramyl-dipeptide. Conditioned media were diluted 1:5.

ap $<0.0001$ (parametric unpaired t-test).

$\mathrm{b}, \mathrm{cP}<0.0022$ (nonparametric Mann-Whitney test). 
liferative capacity of the stromal population, mainly fibroblastic progenitors. In addition, when adherent cells (most of them fibroblasts) from confluent primary bone marrow cultures (13/20) from LCP were further induced to proliferate following four continuous subcultures, reduced or no further growth was observed in seven of them. This defective proliferative potential of the bone marrow fibroblasts in LCP might explain the lower ability of the stromal components to achieve confluence in primary cultures and in the other four subcultures.

The regulation of fibroblast proliferation is tremendously complex. On the one hand, the cellular shape seems to be one of the primary factors that regulate the mitogenic responses of fibroblasts to certain mitogenic agents (18). This becomes evident when one considers cells (such as fibroblasts) in which the proliferative rate is anchorage dependent. When normal bone marrow fibroblasts are plated on plastic, they will spread on the surface and proliferate actively in response to serum factors until reaching confluence (18). And on the other hand, many soluble factors can alter fibroblast growth, as previously reported by our group $(8,12)$.

In the present study we found that pure fibroblasts isolated after four continuous subcultures from bone marrow of LCP released very low levels of IL-1ß $(<10 \mathrm{pg} / \mathrm{ml})$ compared with NC. In contrast, untreated pure fibroblasts from LCP released high levels of PGE2 compared with NC values, and PGE2 production was not increased after MDP stimulation in the LCP group. This result supports the theory that a defective prolif- erative potential of the progeny of CFU-F can be caused by an increase in the production of PGE2. These findings are in agreement with reports by other authors who observed that PGE2 is an inhibitor of fibroblast proliferation in serum-supplemented cultures $(19,20)$.

On the other hand, some authors observed that IL-1ß induces PGE2 in fibroblasts $(9,21)$, whereas our results showed high levels of IL-1ß released by NC subcultures that were not correlated with PGE2 levels, which were lower compared to LCP subcultures that had little or no IL-1ß. Thus, this apparent contradiction suggested that the increased levels of PGE2 in the CM of LCP could be induced by factors other than IL-1 1 , like tumor necrosis factor- $\alpha$ or TGFB $(21,22)$.

By analyzing the results as a whole, we reached the conclusion that bone marrow fibroblasts from LCP had a defective proliferative and confluence capacity not only in the primary cultures but also during the four subsequent subcultures. Moreover, the bone marrow fibroblasts from LCP had an alteration of IL-1ß and PGE2 production that may play an important role, directly or indirectly, in the regulation of the proliferative and confluence capacity of these stromal cells during the subsequent subcultures. Finally, although it seems clear that functional abnormalities in bone marrow fibroblasts exist in LCP, it is not known whether these modifications are an inherent defect in fibroblasts of patients susceptible to lung carcinoma or an acquired defect related to the tumor itself.

\section{References}

1. Castro-Malaspina H, Gay RE, Resnick G, Kapoor N, Meyers P, Chiarieri D, McKenzie S, Broxmeyer HE \& Moore MAS (1980). Characterization of human bone marrow fibroblast colony-forming cells (CFU-F) and their progeny. Blood, 56: 289301.
2. Bentley SA \& Foidart J M (1980). Some properties of marrow derived adherent cells in tissue culture. Blood, 56: 10061012.

3. Krebsbach PH, Kuznetsov SA, Bianco P \& Gehron Robey $P$ (1999). Bone marrow stromal cells: characterization and clinical application. Critical Reviews in Oral Biology and Medicine, 10: 165-181.

4. Moreau I, Andreoni C, Caux C, Saeland S $\&$ Rigal D (1992). Modification of human long-term marrow cultures: Establishment of a functional stromal microenvironment devoid of myeloid progenitors. 
European J ournal of Haematology, 49: 2935.

5. Caplan Al (1994). The mesengenic process. Clinics in Plastic Surgery, 21: 429435.

6. Minguell J J, Conget $P \&$ Erices A (2000). Biology and clinical utilization of mesenchymal progenitor cells. Brazilian J ournal of Medical and Biological Research, 33: 881-887.

7. Chasseing NA, Bordenave RH, Bullorsky EO, Diaz NB, Stemmelin GR \& Rumi L (1997). Fibroblastic colony forming units and the levels of tumor necrosis factor and prostaglandin E2 in bone marrow cultures from patients with advanced lung carcinoma. Cancer, 80: 1914-1919.

8. Kovacs EJ (1991). Fibrogenic cytokines: the role of immune mediators in the development of scar tissue. Immunology Today, 12: 17-23.

9. Freundlich B, Bomalaski J S, Neilson E \& J imenez SA (1986). Regulation of fibroblast proliferation and collagen synthesis by cytokines. Immunology Today, 7: 303307.

10. Thomton SC, Por SB, Walsh BJ , Penny R $\&$ Breit SN (1990). Interaction of immune and connective tissue cells: I. The effect of lymphokines and monokines on fibroblast growth. J ournal of Leukocyte Biology, 47: 312-320.

11. Kuznetsov SA, Friedenstein AJ \& Robey PG (1997). Factors required for bone marrow stromal fibroblast colony formation in-vitro. British J ournal of Haematology, 97: 561-570.

12. Rougier F, Dupuis F \& Denizot $Y$ (1996). Human bone marrow fibroblasts: an overview of their characterization, proliferation and inflammatory mediator production. Hematology and Cell Therapy, 38: 241-246.

13. Known BS, Wang S, Udagawa N, Haridas V, Lee ZH, Kim KK, Oh KO, Greene J , Li Y, Su J , Gentz R, Aggarwal BB \& Ni J (1998). TR1, a new member of the tumor necrosis factor receptor superfamily, induces fibroblast proliferation and inhibits osteoclastogenesis and bone resorption. FASEB J oumal, 12: 845-854.

14. Iribe $\mathrm{H}$, Koga $\mathrm{T}$, Kotani $\mathrm{S}$, Kusumoto $\mathrm{S} \&$ Shiva T (1983). Stimulating effect of muramyl-dipeptide and its adjuvant active analogues on guinea pig fibroblasts for the production of thymocyte-activating factor. J oumal of Experimental Medicine, 157: 2190-2195.

15. Kuznetsov S \& Gehron RP (1996). Species differences in growth requirements for bone marrow stromal fibroblast colony formation in vitro. Calcified Tissue International, 59: 265-270.

16. Coutinho LH, Geary CG, Chang J, Harrison C \& Testa NG (1990). Functional studies of bone marrow haemopoietic and stromal cells in the myelodisplastic syndrome (MDS). British J ournal of Haematology, 75: 16-25.

17. Hirata J, Katsuno M \& Kaneko S (1986).
Clinical significance of human bone marrow stromal cell colonies in acute leukemias. Leukemia Research, 12: 1441-1445.

18. Gospodarowicz D, Greenburg G \& Birdwell CR (1978). Determination of cellular shape by the extracellular matrix and its correlation with the control of cellular growth. Cancer Research, 38: 4155-4171.

19. Hori T, Yamanaka Y, Hayakawa M, Shibamoto $S$, Tsujimoto $\mathrm{M}$, Oku $\mathrm{N} \&$ Ito $\mathrm{F}$ (1991). Prostaglandins antagonize fibroblast proliferation stimulated by tumor necrosis factor. Biochemical and Biophysical Research Communications, 174: 758766.

20. Kom J H, Halushka PV \& LeRoy EC (1980). Mononuclear cell modulation of connective tissue function. Suppression of fibroblast growth by stimulation of endogenous prostaglandin production. J oumal of Clinical Investigation, 65: 543-554.

21. Diaz A, Muñoz E, J ohnston R, Korn J H \& J imenez SA (1993). Regulation of human lung fibroblast $\alpha 1$ (I) procollagen gene expression by tumor necrosis factor $\alpha$, interleukin-1ß, and prostaglandin E2. J ournal of Biological Chemistry, 268: 1036410371.

22. Diaz A, Varga J \& Jimenez SA (1989). Transforming growth factor- $\beta$ stimulation of lung fibroblast prostaglandin E2 production. J ournal of Biological Chemistry, 264: 11554-11557. 